\title{
1 On the use of on-cow accelerometers for the classification of behaviours in
}

\section{2 dairy barns}

3 Said Benaissa ${ }^{a, b}{ }^{*}$, Frank A.M. Tuyttens ${ }^{b, c}$, David Plets ${ }^{a}$, Toon de Pessemier ${ }^{a}$, Jens Trogh ${ }^{a}$, Emmeric Tanghe $^{a}$, Luc Martens ${ }^{a}$, Leen Vandaele ${ }^{b}$, Annelies Van Nuffel ${ }^{d}$, Wout Joseph $^{a}$, Bart Sonck ${ }^{\text {b, e }}$

a Department of Information Technology, Ghent University/imec, iGent-Technologiepark 15, 9052 Ghent, Belgium

${ }^{\mathrm{b}}$ Institute for Agricultural and Fisheries Research (ILVO)- Animal Sciences Unit, Scheldeweg 68, 9090 Melle, Belgium

c Department Nutrition, Genetics and Ethology Laboratory for Ethology Faculty of Veterinary Medicine, D8, Heidestraat 19, B-9820

Merelbeke, Belgium

${ }^{\mathrm{d}}$ Institute for Agricultural and Fisheries Research (ILVO)- Technology and Food Science Unit, Burgemeester van Gansberghelaan 115 bus 1 , 9820 Merelbeke, Belgium

e Department of Biosystems Engineering, Faculty of Bioscience Engineering, Ghent University, Coupure links 653, B-9000 Ghent, Belgium

* Corresponding author. Tel.: +32 0933148 99; fax: +32 093314899 E-mail address: said.benaissa@ugent.be (Said Benaissa)

\section{Abstract}

Analysing behaviours can provide insight into the health and well-being of dairy cows. As herd size increases, automatic monitoring systems based on sensors, such as accelerometers, are becoming increasingly important to accurately quantify cows' behaviours. The aim of this study is to automatically classify cows' behaviours by comparing leg- and neck-mounted accelerometers. In addition, this study investigates the effect of the sampling rate and the number of accelerometer axes logged on the classification performances. Lying, standing, and feeding behaviours of 16 cows were logged for 6 hours with 3D-accelerometers. K-nearest neighbours, naïve Bayes, and support vector machine classification models were constructed based on accelerometers data fitted with the observations made as a reference. Sensitivity, precision, and accuracy were used to evaluate the model performance. 

have classified the three behaviours with high precision (80-99\%) and sensitivity (87-99\%). For the legmounted accelerometer, lying behaviour was classified with high precision (99\%) and sensitivity (98\%). Feeding was classified more accurately by the neck-mounted versus the leg-mounted accelerometer (precision $92 \%$ versus $80 \%$; sensitivity $97 \%$ versus $88 \%$ ). Standing was the most difficult behaviour to classify when only one accelerometer was used. Classification accuracy of cows' behaviours using accelerometers depends on the position of the sensors on the cow's body, the sampling rate, and the number of logged accelerometer axes. A good monitoring system should take into consideration all these parameters in order to minimise the sensors' power consumption while maintaining acceptable performances.

Keywords: Accelerometer, dairy cows, machine learning, behaviors classification, feature extraction.

\section{Introduction}

Changes in behaviours could provide relevant information about nutrition, reproduction, health, and overall well-being of dairy cows. For instance, changes in lying behaviour can indicate underlying shifts in cow comfort and welfare (Ledgerwood et al., 2010; Tucker and Weary, 2004). Several traditional methods such as direct observation of the cows, either live or from video recording, have been used to assess behaviours in dairy farms (Müller and Schrader, 2003). However, due to the time constraints and lack of labour force, especially in large sized farms, progress has been made in monitoring cows with electronic and biosensor devices(Benaissa et al., 2016a, 2016b; Braun et al., 2015; Chapinal et al., 2011; Dutta et al., 2015; Maselyne et al., 2017; Piccione et al., 2011; Van Nuffel et al., 2015). In particular, wearable accelerometers have been widely tested to automatically assess cow behaviours (Martiskainen et al., 2009; Müller and Schrader, 2003; Robert et al., 2009; Vázquez Diosdado et al., 2015). In addition to accelerometers, researchers have proposed the use of various machine learning tools to classify accelerometer data more accurately (Bidder et al., 2014; Langrock et al., 2012; McClune et al., 2014; Resheff et al., 2014). 
For dairy cows, different approaches have been suggested. Robert et al. (2009) used a three-

dimensional leg-mounted accelerometer with a sampling rate of $100 \mathrm{~Hz}$ to monitor and classify three behaviour patterns (i.e., lying, standing, and walking). However, feeding behaviour was not considered in this work. Another study (Mattachini et al., 2013) compared two leg-mounted accelerometer technologies [HOBO Pendant G (Onset Computer Corporation, Pocasset, MA) and IceTag (IceRobotics, Edinburgh, UK)], with video recording to measure lying and standing of dairy cows. The classification was based on the static components of the accelerometer axes, which is impractical in real situations where a slight movement of the cow could change the static components within the same behaviour. A recent study (Vázquez Diosdado et al., 2015) used a simple decision-tree algorithm to detect lying, standing, and feeding behaviours with a neck-mounted accelerometer programmed to log data at $50 \mathrm{~Hz}$. The proposed algorithms required a high sampling rate and also used the static component of the $\mathrm{Y}$-axis to distinguish between standing and lying.

In practice, the sensors use very small batteries with low processing and storage capabilities. Furthermore, such batteries would need to operate properly and autonomously for long periods of time without being recharged or replaced. Therefore, energy consumption is an important issue in using sensors for monitoring behaviour of dairy cows. Several choices can impact energy consumption, e.g., sampling rate, transmit rate, routing methods, and programming languages (Lee and Annavaram, 2012). To reduce the energy consumption and maintenance requirements associated with recharging of batteries while maintaining acceptable performances, choosing the right position of the sensor (e.g., neck or leg), working with lower sampling rate, or logging fewer accelerometer axes are important considerations. In this study, a relatively low sampling rate $(1 \mathrm{~Hz})$ and parameters derived from the three axes were used. Also, to the best of our knowledge, no study has compared leg- and neckmounted accelerometers and investigated the effect of the sampling rate and the number of axes logged by the accelerometer ( $\mathrm{X}, \mathrm{Y}$, and $\mathrm{Z}$ ) on the accuracy of behavioural classification. 
The aim of this study is to automatically classify cows' behaviours (i.e., lying, standing, and feeding) based on machine learning algorithms (i.e., K-nearest neighbours, naïve Bayes, and Support Vector Machine) (Martiskainen et al., 2009; Vázquez Diosdado et al., 2015) by comparing leg- and neckmounted accelerometers. Additionally, since cow-mounted measuring devices are energy- and memory-constrained, we investigated the effect of decreasing the sampling rate and reducing the number of accelerometer axes logged on the classification performances of the developed automatic classification system.

\section{Materials and methods}

\subsection{Animal and housing}

Measurements were conducted between March and July 2016 in a dairy cattle research barn of the Flemish research institute for agriculture, fisheries and food (ILVO) in Melle, Belgium. From a group of 31 cows, 16 different second parity Holstein cows (milk yield $33.6 \pm 5.6 \mathrm{~kg} / \mathrm{d}$; mean \pm SD) were used for this study. The cows were housed in an area of $30 \mathrm{~m}$ long and $13 \mathrm{~m}$ wide with individual cubicles and concrete slatted floor. The cubicles $(n=32$, width $115 \mathrm{~cm}$, length from curb to front rail $178 \mathrm{~cm}$, front rail height $70 \mathrm{~cm}$, neck rail height $109 \mathrm{~cm}$, neck rail distance from curb $168 \mathrm{~cm}$ ) were bedded with a lime-straw-water mixture. The cows had access to a milking robot via the feeding area and a smart selection gate in a feed-first cow traffic system. A cow was allowed access to the milking robot based on different parameters such as the interval since the previous milking, expected milk yield, and lactation stage. The cows were fed roughage ad libitum and the amount of protein rich and balanced concentrate was fixed depending on lactation stage and production level. The concentrates were supplied both in the milking robot and by computerized concentrate feeders. Drinking water was available ad libitum. The cows had free access to a rotating cow brush.

\subsection{Behaviours' observation}

Two cows were monitored simultaneously from 10 AM to 4 PM as the sensors' memory could not save more than 6 hours of the data. Observations on the behaviour of the cows were made directly in the 
barn by a student and with video recordings at the same time as data from the sensors were collected. Table 1 lists the considered behaviours in this study with their descriptive definitions. The video recordings were taken as a secondary measure to ensure that all behavioural data was captured during the observation period. Around $90 \%$ of the data were labelled just by the direct observation while $10 \%$ of the data were labelled based on the video recordings, when the direct observation of the cows was difficult.

The methodology of the observation was as follows. Every minute time window was assigned with a label to refer to lying, standing, and feeding behaviours, respectively, based on the behaviour that was present during the largest proportion of that minute. Instead of removing the small number of samples of the drinking behaviour, they were considered as feeding. Similarly, walking was considered as standing. We note that walking was not considered as a separate behaviour, because it was observed less frequently and for shorter durations (on average, 8 to 12 minutes per cow).

\subsection{Accelerometer data}

Two accelerometers were attached to each cow. The first accelerometer was attached to the neck collar (right side) and the second was attached to the right hind leg as shown in Figure 1 . The acceleration data were logged with a sampling rate of $1 \mathrm{~Hz}$ (1 sample each second) using HOBO loggers (Onset Computer Corporation, Pocasset, MA). The HOBO logger is a waterproof 3-channel logger with 8-bit resolution, which can record up to approximately 21,800 combined acceleration readings or internal logger events. The logger uses an internal 3 -axis accelerometer with a range of $\pm 3 \mathrm{~g}$ (accuracy $\pm 0.075 \mathrm{~g}$ at $25^{\circ} \mathrm{C}$ with a resolution of $0.025 \mathrm{~g}$ ) based on micro-machined silicon sensors consisting of beams that deflect with acceleration.

The orientation of the accelerometers when the cow is standing and lying is shown in Figure 1. This orientation was respected for all cows. The clocks of the observer, the video recording system, and the sensors were synchronized at the start and at the end of the observation period so that observation data could be aligned accurately with the tri-axial accelerometer data retrieved from the sensors. In 
total, 96 hours of data (i.e., $6 \mathrm{~h} / \mathrm{cow}, 16$ cows total) were recorded for every accelerometer and used for classification of the behaviours.

\subsection{Data pre-processing}

A summary of the data processing and classification procedure is shown in figure 2 . First, the sensor data were downloaded from the accelerometer using Onset HOBOware software version 3.7.5 (Onset Computer Corp.). These data were exported into .csv files. Then, Octave software was designed to segment the data into equal time intervals of $1 \mathrm{~min}$ (60 samples) and to extract the features (e.g., mean, max) for each time interval. Next, based on the observations of the cows' behaviours, behaviour labels vectors were constructed. These vectors (reference data) and the calculated feature vectors (sensor data) were used as an input to the classification algorithms. Finally, a validation of the developed behaviour classifiers was performed by measuring their performances in terms of precision, sensitivity, and the overall accuracy.

Raw time series collected from 16 individual cows and uploaded to the laptop were pre-processed first using HOBOware software. The data were exported to .csv files (32 files). From the accelerations along $\mathrm{X}, \mathrm{Y}$, and $\mathrm{Z}$ axes, the acceleration sum vector $\left(A_{\text {sum }}\right)$ was calculated as follows:

$$
A_{\text {sum }}=\sqrt{a_{X}^{2}+a_{Y}^{2}+a_{Z}^{2}}
$$

Where, $a_{X}$ is the acceleration along the $\mathrm{X}$-axis, $a_{Y}$ is the acceleration along the $\mathrm{Y}$-axis, and $a_{Z}$ is the acceleration along the Z-axis. The sum vector was added to the .csv files in parallel to the individual accelerations along the three axes. Figure 3 shows an example of the time series acceleration sum vector $\left(A_{\text {sum }}\right)$ obtained from leg and neck accelerometers. For both sensors, when a cow is feeding, large variations were registered in comparison with standing and lying. This is an important characteristic that is exploited in the feature extraction phase (Section 2.5). 
After the pre-processing of the sensor data and obtaining the .csv files, Octave software was used to segment the sensor data to equal time intervals of $1 \mathrm{~min}$. Features extraction is then performed for each data segment to transform the input data into a representation set of features, also referred to as feature vectors (Avci et al., 2010). Feature vectors include important parameters for distinguishing various behaviours and they are then used as input to the developed classification algorithms.

In this study, time- and frequency-domain features were used. Time-domain features are directly derived from the time-dependent raw acceleration data for each time interval. These features include basic signal statistics (e.g., mean, standard deviation...) and other waveform characteristics (e.g., dynamic acceleration). Frequency-domain features (e.g., spectral energy) include the periodic characteristics of the signal, such as coefficients derived from Fourier transforms.

\subsubsection{Statistical features}

Eight statistical features were derived directly from the sum vector $\left(A_{\text {sum }}\right)$ for each 1 min time interval (60 samples): minimum, first quartile, median, third quartile, maximum, mean, root mean square, and standard deviation.

\subsubsection{Overall dynamic body acceleration}

To isolate the components caused directly by the movement of the animal, the overall dynamic body acceleration (ODBA) and its vectorial variation (VeDBA) were used in this study. The ODBA and the VeDBA quantify the three-dimensional movement of animals as the value of acceleration and are assumed to be proxies for activity-specific energy expenditure (Wilson et al., 2006).

To calculate the $O D B A$ and $V e D B A$, the time series accelerometer data are converted first to $D B A$. $D B A_{i}(k)$ at any point in time $k$ (each second) is obtained by smoothing each axis $a_{i}(i=X, Y, Z)$ using a running mean $\mu_{i}$ of 5 seconds as in Vázquez Diosdado et al. (2015) to derive the static acceleration and then subtracting this static acceleration from the raw data as follows (Gleiss et al., 2011): 


$$
D B A_{i}(k)=\left|a_{i}(k)-\mu_{i}\right|
$$

171

176

177

179

180

181

182

These values for $D B A$ are then summed to provide $O D B A$ and its vectorial sum $V e D B A$ :

$$
\begin{gathered}
O D B A=D B A_{X}+D B A_{Y}+D B A_{Z} \\
V e D B A=\sqrt{D B{A_{X}}^{2}+D B{A_{Y}}^{2}+D B A_{Z}^{2}}
\end{gathered}
$$

The values of ODBA and VeDBA are given for each 1 second. Then, their statistical features (minimum, first quartile, median, etc.) for each 1 min are calculated as performed for the acceleration sum vector $\left(A_{\text {sum }}\right)$.

\subsubsection{Spectral energy}

The spectral energy feature is the sum of the squared discrete Fast Fourier Transform (FFT) component magnitudes of the signal. The sum is divided by the window length $N$ (60 samples) for normalization. The spectral energy is equal to the energy of the signal (from Parseval's Theorem).

\subsubsection{Spectral entropy}

The spectral entropy was used in (Wang et al., 2005) to discriminate the behaviours with similar energy values (e.g., lying and standing). To calculate the spectral entropy for each 1 min time interval, the normalized power spectral density $p_{k}$ is computed form the FFT components $A(1), A(2), \ldots A(N=$ 60) using the following equation:

$$
p_{k}=\frac{|A(k)|^{2}}{\sum_{k=1}^{N=60}|A(k)|^{2}}
$$

By definition, the mathematical formulation of the spectral entropy is given by:

$$
\text { Spectral_Entropy }=\sum_{k=1}^{N=60} p_{k} \log _{2}\left(p_{k}\right)
$$

In conclusion, for each 1 min time interval containing 60 samples, 26 features were calculated. Eight statistical features of the sum vector $\left(A_{\text {sum }}\right)$, the ODBA, and the VeDBA, in addition to the spectral energy, and the spectral entropy. 
193 In this study, three supervised machine learning algorithms were used for behaviour classification: K-

194 nearest neighbours (Browne, 2000), naïve Bayes, and support vector machine (Sellers and Crompton, 195 2004). A supervised learning algorithm is formed by two processes: training and testing. It uses a 196 known data set to construct a model (training process) that is then used for making predictions on a 197 new data set (testing process). The supervised learning is preferable when the 'categories' or 'classes' 198 are known (for example in this case, standing, lying, feeding). However, in unsupervised learning, the 199 classes are unknown, and the learning process attempts to find appropriate classes. The K-nearest 200 neighbours and the naive Bayes classifiers are possible options because they are fast, simple and well 201 understood (Frank et al., 2000). Regarding the support vector machine (SVM), it can handle better complex classification tasks, but it requires more computational costs, especially in the training phase (Bishop, 2006). To make a fair comparison, the same datasets (number of samples and features) were used as input to the considered algorithms.

\subsection{Performance evaluation}

To measure the performances of the classification approaches, the precision, the sensitivity, and the overall accuracy were used. Since data were collected on 16 cows, the leave one out cross validation strategy was used (Arlot and Celisse, 2010). Therefore, data collected on 15 cows was used to train the system and then the system was tested by classifying the data of the sixteenth cow accordingly. This was repeated 16 times until data from all the cows was classified and the average precision, sensitivity and overall accuracy were considered (Section 3). The precision ( $\mathrm{Pr}$ ) and the sensitivity (Se) are defined as (Chawla, 2005):

$$
\begin{aligned}
& P r=\frac{T P}{T P+F P} \\
& S e=\frac{T P}{T P+F N}
\end{aligned}
$$

Here, TP (true positive) is the number of instances where the behaviour was correctly classified by the algorithm using observations as reference. FN (false negative) is the number of instances where the 
behaviour was visually observed but was incorrectly classified by the algorithm. FP (false positive) is the number of times the behaviour was incorrectly classified by the algorithm based on the reference.

The overall model accuracy is the number of TP instances of all behavioural classes divided by the total number of instances in the test set.

\subsection{Effects of reducing the number of axes and the sampling rate}

To study the effects of reducing the number of the accelerometer axes on the classification accuracy, the features presented in Section 2.5 were calculated again using one axis (e.g., X-axis) or two axes (e.g., XZ-axes) instead of three axes and used as an input for the classification algorithms.

For the effect of the sampling rate on the classification accuracy, the complete data set exported with HOBOware was resampled using Octave software at four different sampling rates (i.e., $0.05 \mathrm{~Hz}, 0.1 \mathrm{~Hz}$, $0.25 \mathrm{~Hz}$, and $0.5 \mathrm{~Hz}$ ). Then, the features presented in Section 2.5 were computed for each sampling rate and the considered algorithms presented in Section 2.6 were used for the classification.

\section{Results}

\subsection{Neck and leg accelerometers combined}

The precision and sensitivity of the considered behaviours and classification algorithms when the features extracted from leg- and neck-mounted accelerometers were combined and used for the classification are listed in Table 2 (column 1). The precision and sensitivity were excellent for the three behavioural classes and the three algorithms with values between $80 \%$ and $99 \%$ for the precision and $87 \%$ and $99 \%$ for the sensitivity. Consequently, high overall accuracy was obtained with values between $93 \%$ and $98 \%$ (Table 3).

\subsection{Leg- versus neck-mounted accelerometers}

The precision and sensitivity using leg-mounted accelerometer with thee axes (XYZ) were high (>93\%) for all algorithms for lying behaviour (Table 2). The precision and sensitivity of feeding behaviour were reasonable with values between 72\% (Naïve Bayes) and 86\% (SVM). Accuracy of classifying standing 
behaviour was lowest, with maximum precision and sensitivity of $76 \%$ and $68 \%$, respectively. The best

242 classification accuracy was obtained using the SVM algorithm (88\%), followed by the K-NN (84\%) and

243 Naïve Bayes (83\%) (Table 3).

244

Unlike the leg-mounted accelerometer, feeding was the best classified behaviour by the neck-mounted accelerometer data with a sensitivity between $95 \%$ and $98 \%$ and a precision between $88 \%$ and $92 \%$ (Table 2). Similar to the leg-mounted accelerometer, standing was the most difficult behaviour to classify with a sensitivity lower than $65 \%$ for all classifiers. For the overall accuracy, SVM was the best classifier followed by K-NN and Naïve Bayes as was also the case for the leg-mounted accelerometer (Table 3). The overall accuracy was slightly higher for the neck-mounted accelerometer than the legmounted accelerometers.

\subsection{Effect of number of accelerometer axes on the classification accuracy}

For the three cases (neck, leg, and neck + leg), the performances were not highly decreased by using one or two axes in comparison to three axes, especially for lying behaviour (Table 2). When data from the neck- and leg-mounted accelerometers were combined, classification of the three behaviours improved for both the X-axis alone (Pr 89-99\%; Se 88-100\%; accuracy 96-97\%) and the Y- and X-axes (Pr 91-99\%; Se 87-100\%, accuracy 97-99\%) compared to XYZ-axes ( $\operatorname{Pr} 80-99 \%$; Se 86-99\%, accuracy 9398\%). Results of XZ-axes were comparable to XYZ for the three behaviours. Moreover, both lying and feeding behaviours were accurately classified with either Y-axis ( $\operatorname{Pr} 85-95 \%$; Se 88-96\%), Z-axis ( $\operatorname{Pr} 80$ 94\%; Se 89-95\%), and XY-axes ( $\operatorname{Pr} 76-95 \%$; Se 86-97\%). However, with these axis configurations, standing was still difficult to classify even with two accelerometers ( $\operatorname{Pr} 55-83 \%$; Se 50-76\%).

When using only the X-axis of the leg-mounted accelerometer, lying behaviour was classified with high precision and sensitivity (Se and $\operatorname{Pr}$ between 97\% and 100\%). In addition, for the neck-mounted accelerometer, both feeding and lying were accurately classified with either one or two axes. The precision and sensitivity varied from $82 \%$ to $97 \%$ and from $78 \%$ to $98 \%$ for feeding and lying behaviours, respectively. The overall accuracy varied between $75 \%$ and $86 \%$ by using $\mathrm{X}-$, XZ-, or YZ- 
axes of the leg-mounted accelerometer and between $76 \%$ and $85 \%$ for all axes configurations of the neck-mounted accelerometer.

\subsection{Effect of sampling rate on the classification accuracy}

As expected, the accuracy decreased for lower sampling rates (Fig. 4). The Naïve Bayes algorithms was influenced most by the decrease of the sampling rate especially for the leg-mounted accelerometer and with sampling rates below $0.25 \mathrm{~Hz}$ (Fig. 4). However, for both leg- and neck-mounted accelerometers, the classification accuracy was still over $80 \%$ for SVM algorithm when $0.25 \mathrm{~Hz}$ was used (1 sample every 4 seconds).

\section{Discussion}

We investigated the performance of classifying three behaviours from data obtained from accelerometers worn by dairy cattle. As expected, the best classification performances were obtained with the set-up in which most data was used, i.e. using both accelerometers, the three axes, and the highest sampling rate $(1 \mathrm{~Hz})$. However, when only one sensor was used for the classification, two behaviours were often confused with each other: standing and feeding in the case of the leg-mounted accelerometer, and standing and lying in the case of the neck-mounted accelerometer. The neck of the cow shows high activity during feeding, which explains why neck-mounted accelerometer data allow this behaviour to be distinguished easily from the other two behaviours (Martiskainen et al., 2009). However, the neck generally moves little during both standing and lying, which makes it hard to differentiate these two behaviours based on the neck-mounted accelerometer. Lying time was more accurately measured by the leg-mounted accelerometer (sensitivity around $100 \%$ ), possibly due to the smaller amount of position changes that the cow's legs make when she is lying. However, the legs have similar patterns most of the time during standing and feeding behaviours, which results in a frequent misclassification of these behaviours. Thus, the best position for an accelerometer depends on the behaviour of interest. Similar conclusions were also drawn by (Martiskainen et al., 2009) and (Mattachini et al., 2013). In (Martiskainen et al., 2009), a neck-mounted accelerometer with a sampling 
rate of $10 \mathrm{~Hz}$ was used to classify cows' behaviours based on the SVM algorithm. In their study, standing and lying behaviours were confused with each other in $30 \%$ of the cases and feeding was misclassified as standing in $14 \%$ of the cases. In the study by Mattachini et al. (2013), lying behaviour was reported as the easiest behaviour to classify with a sensitivity of $98 \%$ using leg-mounted accelerometers (IceTag or HOBO accelerometers). Consequently, the position where the accelerometer is attached on the cow might depend on the goal of the system. Neck mounted accelerometers are better suited for monitoring feeding patterns, leg-mounted accelerometers if highly accurate classification of lying behaviour is needed, and both positions if high accuracy of all three behaviours is needed.

In general, the SVM algorithm performed better than the other algorithms (Alpaydın, 2014). The SVM algorithm is more suitable for complex classification tasks and it requires more computation capabilities than Naïve Bays and K-NN (Douglas et al., 2011), especially in the training phase. However, after the classification model is developed, the SVM classifies the new data without looking to the training set, which would save the memory of the monitoring system, in contrast to the Naïve Bays and the K-NN, where the training set is always required to classify the new instances (Goodfellow et al., 2016). Therefore, the selection of the best classification algorithms is a trade-off between performance and computation/memory capabilities.

As the next step, the number of axes logged by the accelerometers was investigated. With two accelerometers working simultaneously (combination of leg and neck), the classification performances were a little bit higher with $\mathrm{X}$-axis alone or $\mathrm{YZ}$-axes compared to the three axes together. This means that reducing the number of axes logged by the accelerometers would not only minimize the power consumption and data load, but it could also enhance the performances of the classification algorithms. Moreover, the results of the other axis configurations (e.g., $\mathrm{XY}$ and $\mathrm{Y}$ ) were in general comparable to the results of three axes configuration. Consequently, optimizing the number of axes seems possible when the combination of the two sensors is used for the classification. 
In contrast to the results of the combination of leg- and neck-mounted accelerometers, when one accelerometer was used, the reduction of the number of axes decreased the overall accuracy. However, individual behaviours were perfectly classified with fewer axes (e.g., lying behaviour with the $\mathrm{X}$-axis of the leg-mounted accelerometer and feeding behaviour with YZ-axes of the neck-mounted accelerometer). Lying behaviour was perfectly classified with the X-axis of the leg-mounted accelerometer because after the transition from lying to standing, this axis becomes horizontal (variations around $1 \mathrm{~m} / \mathrm{s}^{2}$ ) instead of perpendicular to the ground (variations around $0 \mathrm{~m} / \mathrm{s}^{2}$ ). This means that if the user is mainly interested in long-term monitoring of the lying behaviour of the herd, programming a leg-mounted accelerometer to log only X-axis can be recommended. These findings are in agreement with the results of (Ledgerwood et al., 2010), where one axis (Y-axis) of a legmounted accelerometer was used to record lying behaviour.

The use of one axis instead of three axes for classifying behaviours has also been investigated by (Ito et al., 2009). In their study, the degree of the vertical tilt (X-axis) from a leg-mounted accelerometer was used to determine the lying behaviour of the cows. In addition, (Mattachini et al., 2013) used the degree of Z-axis tilt to determine the laterality of lying behaviour (right or left side). Although one axis was used for the classification in these studies, only lying behaviour was considered. Also, the method proposed was limited to leg-mounted accelerometers and cannot be used for neck-mounted accelerometers.

The last step was the investigation of the sampling rate. The accuracy decreased for lower sampling rates for both accelerometers. However, it was still over $80 \%$ for the SVM algorithm when $0.25 \mathrm{~Hz}$ was used (1 sample every 4 seconds). Such a considerable reduction in sampling rate could save the sensors' power and minimise the storage load of the monitoring system (a reduction of $75 \%$ ). The decrease in the ability of accelerometers to identify locomotion behaviour patterns when the sampling rate decreases was also remarked when monitoring goat behaviours (Moreau et al., 2009). To overcome this decrease, an appropriate selection of the classification algorithm could enhance the 
accuracy when lower sampling rates are used. However, the sampling rate should not be lower than $0.01 \mathrm{~Hz}$ if the farmer is interested in measuring other aspects of lying behaviour (e.g., lying bouts) as reported by (Mattachini et al., 2013).

More data would be needed especially from other herds to validate the findings of this research. Furthermore, the selection of relevant features should also be addressed in order to reduce the number of features used for the classification. This would lower the computation time of the algorithms as well as enhance their performances. Finally, the data logging time per cow (i.e., 6 hours) was not sufficient to collect enough data for some behaviours such as walking and drinking. These behaviours could be set in separate behavioural classes when many more samples would be available.

\section{Conclusions and future work}

In this paper, leg- and neck-mounted accelerometers have been used for the classification of dairy cows' behaviours. Also, the effects of the sampling rate and the number of accelerometers axes on the classification accuracy have been investigated. Results have shown that the classification performance of cows' behaviours using accelerometers depends on the position of the sensors on the cow's body, the sampling rate, and the number of logged accelerometer axes. A good monitoring system should take into consideration all these parameters in order to minimise the sensors' power consumption, while maintaining a reasonable classification accuracy. Future work will consist of expanding this research to other herds, additional behaviours (ruminating, grooming), and different environments (e.g., pasture), in order to broaden the possible applications of the monitoring system. This would enable the determination of relevant information about the cows' behaviour patterns (e.g., feeding time, lying time, lying bouts). Such information could offer new potential technologies for the automated detection of health and welfare problems in dairy cows. 


\section{Acknowledgments}

This work was executed within MoniCow, a research project bringing together academic researchers and industry partners. The MoniCow project was co-financed by imec (iMinds) and received project support from Flanders Innovation \& Entrepreneurship. E. Tanghe is a Postdoctoral Fellow of the FWOV (Research Foundation - Flanders). The authors would like to thank Thijs Decroos and Sara Van Lembergen for their help during the measurements.

\section{References}

Alpaydın, E., 2014. Introduction to machine learning, Methods in Molecular Biology. doi:10.1007/978-1-62703-748-8-7

Arlot, S., Celisse, A., 2010. A survey of cross-validation procedures for model selection. Stat. Surv. 4, 40-79. doi:10.1214/09-SS054

Avci, A., Bosch, S., Marin-Perianu, M., Marin-Perianu, R., Havinga, P., 2010. Activity Recognition Using Inertial Sensing for Healthcare, Wellbeing and Sports Applications: A Survey. Archit. Comput. Syst. (ARCS), 2010 23rd Int. Conf. 1-10.

Benaissa, S., Plets, D., Tanghe, E., Verloock, L., Martens, L., Hoebeke, J., Sonck, B., Tuyttens, F.A.M., Vandaele, L., Stevens, N., Joseph, W., 2016a. Experimental characterisation of the off-body wireless channel at $2.4 \mathrm{GHz}$ for dairy cows in barns and pastures. Comput. Electron. Agric. 127, 593-605. doi:10.1016/j.compag.2016.07.026

Benaissa, S., Plets, D., Tanghe, E., Vermeeren, G., Martens, L., Sonck, B., Tuyttens, F.A.M., Vandaele, L., Hoebeke, J., Stevens, N., Joseph, W., 2016b. Characterization of the on-body path loss at 2.45 GHz and energy efficient WBAN design for dairy cows. IEEE Trans. Antennas Propag. 11, 48484858. doi:10.1109/TAP.2016.2606571

Bidder, O.R., Campbell, H.A., Gómez-Laich, A., Urgé, P., Walker, J., Cai, Y., Gao, L., Quintana, F., Wilson, R.P., 2014. Love thy neighbour: Automatic animal behavioural classification of acceleration data using the k-nearest neighbour algorithm. PLoS One 9. doi:10.1371/journal.pone.0088609

Bishop, C.M., 2006. Pattern Recognition and Machine Learning, Pattern Recognition. doi:10.1117/1.2819119 
Braun, U., Zürcher, S., Hässig, M., 2015. Eating and rumination activity in 10 cows over 10 days. Res. Vet. Sci. 101, 196-198. doi:10.1016/j.rvsc.2015.05.001

Browne, M., 2000. Cross-Validation Methods. J. Math. Psychol. 44, 108-132. doi:10.1006/jmps.1999.1279

Chapinal, N., de Passillé, A., Pastell, M., Hänninen, L., Munksgaard, L., Rushen, J., 2011. Measurement of acceleration while walking as an automated method for gait assessment in dairy cattle. J. Dairy Sci. 94, 2895-2901. doi:10.3168/jds.2010-3882

Chawla, N. V, 2005. Data Mining for Imbalanced Datasets: An Overview. Data Min. Knowl. Discov. Handb. 853-867. doi:10.1007/0-387-25465-X_40

Douglas, P.K., Harris, S., Yuille, A., Cohen, M.S., 2011. Performance comparison of machine learning algorithms and number of independent components used in fMRI decoding of belief vs. disbelief. Neuroimage 56, 544-553. doi:10.1016/j.neuroimage.2010.11.002

Dutta, R., Smith, D., Rawnsley, R., Bishop-Hurley, G., Hills, J., Timms, G., Henry, D., 2015. Dynamic cattle behavioural classification using supervised ensemble classifiers. Comput. Electron. Agric. 111, 18-28. doi:10.1016/j.compag.2014.12.002

Frank, E., Trigg, L., Holmes, G., Witten, I.H., 2000. Naive Bayes for Regression. Mach. Learn. doi:10.1023/A:1007670802811

Gleiss, A.C., Wilson, R.P., Shepard, E.L.C., 2011. Making overall dynamic body acceleration work: On the theory of acceleration as a proxy for energy expenditure. Methods Ecol. Evol. 2, 23-33. doi:10.1111/j.2041-210X.2010.00057.x

Goodfellow, I., Bengio, Y., Courville, A., 2016. Machine Learning Basics. Deep Learn. 94-159. doi:10.1533/9780857099440.59

Ito, K., Weary, D.M., von Keyserlingk, M.A.G., 2009. Lying behavior: Assessing within- and betweenherd variation in free-stall-housed dairy cows. J. Dairy Sci. 92, 4412-4420. doi:10.3168/jds.2009-2235

Langrock, R., King, R., Matthiopoulos, J., Thomas, L., Fortin, D., Morales, J.M., 2012. Flexible and practical modeling of animal telemetry data: Hidden Markov models and extensions. Ecology 93, 2336-2342. doi:10.1890/11-2241.1

Ledgerwood, D.N., Winckler, C., Tucker, C.B., 2010. Evaluation of data loggers, sampling intervals, and editing techniques for measuring the lying behavior of dairy cattle. J. Dairy Sci. 93, 5129-39. doi:10.3168/jds.2009-2945 
Lee, S., Annavaram, M., 2012. Wireless Body Area Networks: Where does energy go?, in: 2012 IEEE International Symposium on Workload Characterization (IISWC). pp. 25-35. doi:10.1109/IISWC.2012.6402897

Martiskainen, P., Järvinen, M., Skön, J.-P., Tiirikainen, J., Kolehmainen, M., Mononen, J., 2009. Cow behaviour pattern recognition using a three-dimensional accelerometer and support vector machines. Appl. Anim. Behav. Sci. 119, 32-38. doi:10.1016/j.applanim.2009.03.005

Maselyne, J., Pastell, M., Thomsen, P.T., Thorup, V.M., H??nninen, L., Vangeyte, J., Van Nuffel, A., Munksgaard, L., 2017. Daily lying time, motion index and step frequency in dairy cows change throughout lactation. Res. Vet. Sci. 110, 01-03. doi:10.1016/j.rvsc.2016.10.003

Mattachini, G., Riva, E., Bisaglia, C., Pompe, J.C.A.M., Provolo, G., 2013. Methodology for quantifying the behavioral activity of dairy cows in freestall barns. J. Anim. Sci. 91, 4899-4907. doi:10.2527/jas2012-5554

McClune, D.W., Marks, N.J., Wilson, R.P., Houghton, J.D., Montgomery, I.W., McGowan, N.E., Gormley, E., Scantlebury, M., 2014. Tri-axial accelerometers quantify behaviour in the Eurasian badger (Meles meles): towards an automated interpretation of field data. Anim. Biotelemetry 2, 5. doi:10.1186/2050-3385-2-5

Moreau, M., Siebert, S., Buerkert, A., Schlecht, E., 2009. Use of a tri-axial accelerometer for automated recording and classification of goats' grazing behaviour. Appl. Anim. Behav. Sci. 119, 158-170. doi:10.1016/j.applanim.2009.04.008

Müller, R., Schrader, L., 2003. A new method to measure behavioural activity levels in dairy cows. Appl. Anim. Behav. Sci. 83, 247-258. doi:10.1016/S0168-1591(03)00141-2

Piccione, G., Giannetto, C., Schembari, A., Gianesella, M., Morgante, M., 2011. A comparison of daily total locomotor activity between the lactation and the dry period in dairy cattle. Res. Vet. Sci. 91, 289-293. doi:10.1016/j.rvsc.2010.12.011

Resheff, Y.S., Rotics, S., Harel, R., Spiegel, O., Nathan, R., 2014. AcceleRater: a web application for supervised learning of behavioral modes from acceleration measurements. Mov. Ecol. 2, 27. doi:10.1186/s40462-014-0027-0

Robert, B., White, B.J., Renter, D.G., Larson, R.L., 2009. Evaluation of three-dimensional accelerometers to monitor and classify behavior patterns in cattle. Comput. Electron. Agric. 67, 80-84. doi:10.1016/j.compag.2009.03.002

Sellers, W.I., Crompton, R.H., 2004. Automatic monitoring of primate locomotor behaviour using 
Tucker, C.B., Weary, D.M., 2004. Bedding on geotextile mattresses: how much is needed to improve cow comfort? J. Dairy Sci. 87, 2889-2895. doi:10.3168/jds.S0022-0302(04)73419-0

459

460

461

462

463

464

465

466

467

468

469

470

471

472

Van Nuffel, A., Zwertvaegher, I., Van Weyenberg, S., Pastell, M., Thorup, V.M., Bahr, C., Sonck, B., Saeys, W., 2015. Lameness detection in dairy cows: Part 2. Use of sensors to automatically register changes in locomotion or behavior. Animals. doi:10.3390/ani5030388

Vázquez Diosdado, J.A., Barker, Z.E., Hodges, H.R., Amory, J.R., Croft, D.P., Bell, N.J., Codling, E.A., 2015. Classification of behaviour in housed dairy cows using an accelerometer-based activity monitoring system. Anim. Biotelemetry 3, 15. doi:10.1186/s40317-015-0045-8

Wang, S.W.S., Yang, J.Y.J., Chen, N.C.N., Chen, X.C.X., Zhang, Q.Z.Q., 2005. Human activity recognition with user-free accelerometers in the sensor networks. 2005 Int. Conf. Neural Networks Brain 2, 1212-1217. doi:10.1109/ICNNB.2005.1614831

Wilson, R.P., White, C.R., Quintana, F., Halsey, L.G., Liebsch, N., Martin, G.R., Butler, P.J., 2006. Moving towards acceleration for estimates of activity-specific metabolic rate in free-living animals: The case of the cormorant. J. Anim. Ecol. 75, 1081-1090. doi:10.1111/j.13652656.2006.01127.x 
Fig. 1. Position and orientation of the accelerometers when the cow is standing (a) and lying (b). Close-up view of the neck- (c) and the leg-mounted (d) acceleromters.

a)

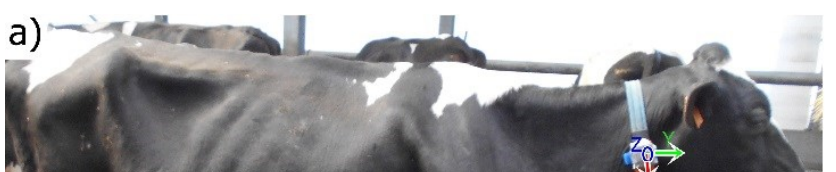

b)

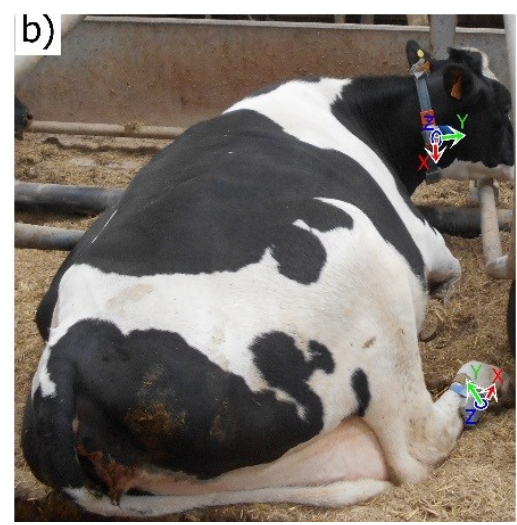

c)

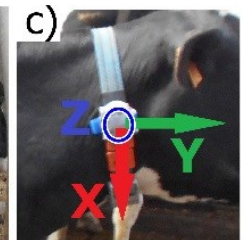

d)

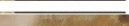

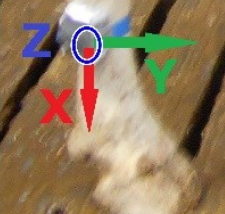

477

478

479

480

481

482

483

484

485

486

487

488

489

490

491

492 
Fig. 2. Data processing and classification procedure.

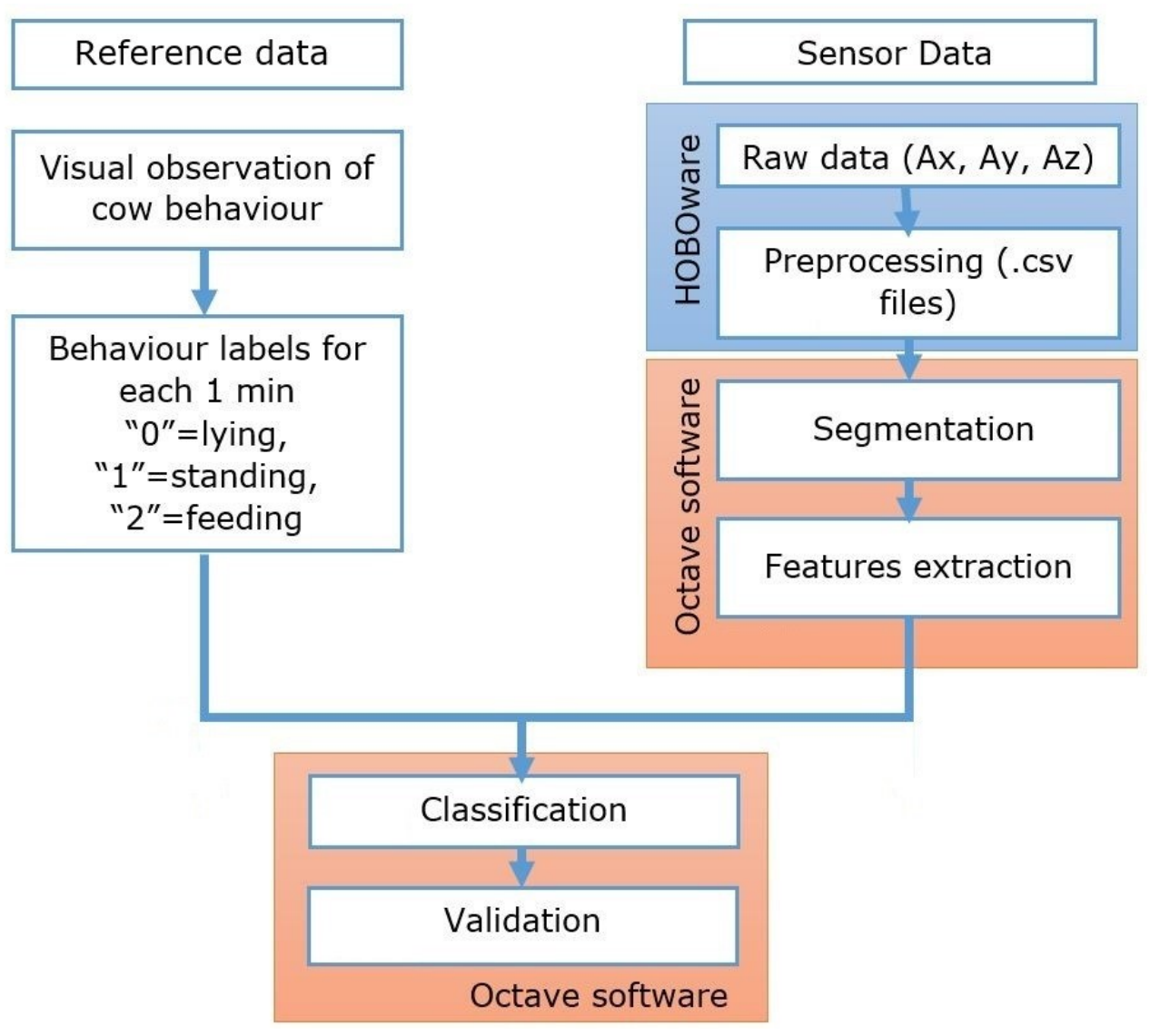

495

496

497

498

499

500

501

502

503

504 
Fig. 3. Example of the acceleration sum vector $\left(A_{\text {sum }}\right)$ from leg- and neck-mounted accelerometers for the considered behaviours.
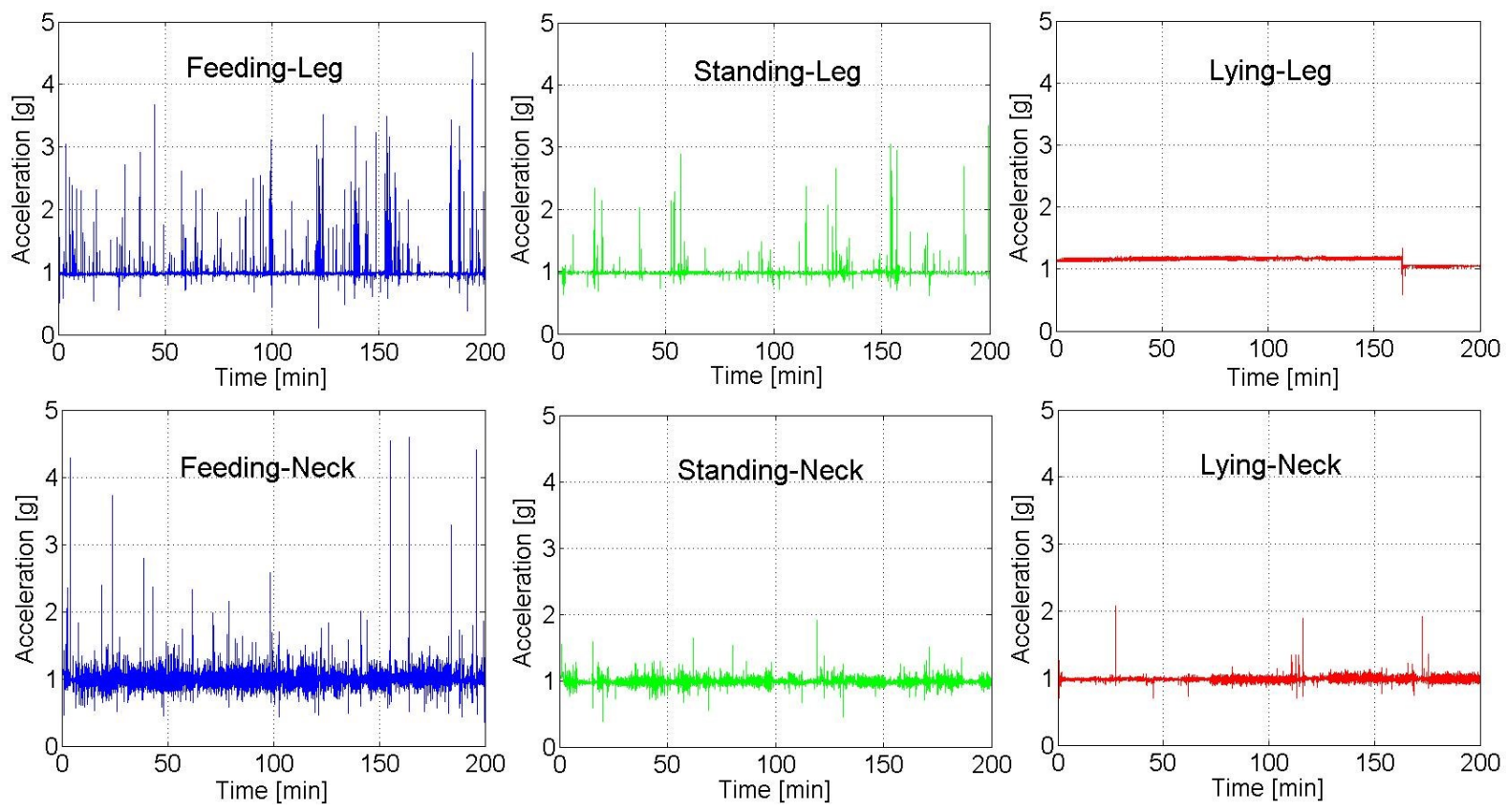

508

509

510

511

512

513

514

515

516

517

518

519

520 
Fig. 4. Classification accuracy as a function of the sampling rate for the leg- and neck-mounted accelerometers.

(a) Leg accelerometer

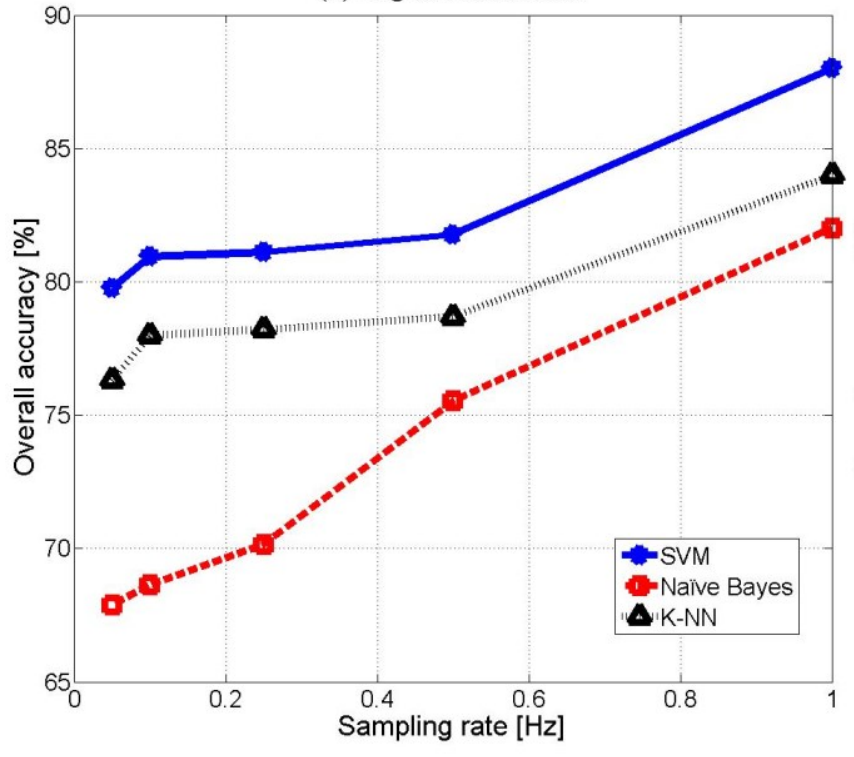

(b) Neck accelerometer

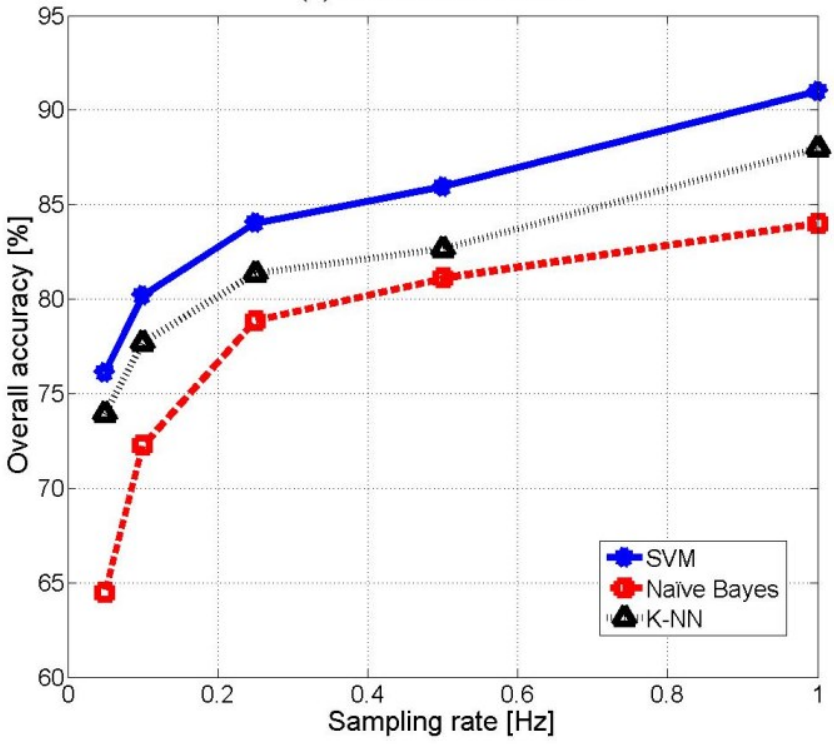

523 
526 Table 1. Description of the observed behaviours. The behaviours are grouped in three behavioural 527 classes (i.e., feeding, standing, lying)

\begin{tabular}{|c|c|c|c|c|}
\hline $\begin{array}{l}\text { Observed } \\
\text { Behaviours }\end{array}$ & Description & $\begin{array}{l}\text { Number of } \\
\text { samples* }\end{array}$ & $\begin{array}{l}\text { Behavioural } \\
\text { class }\end{array}$ & $\begin{array}{l}\text { Total Number } \\
\text { of samples** }\end{array}$ \\
\hline $\begin{array}{l}\text { Feeding pattern } \\
\text { at feed bunk }\end{array}$ & $\begin{array}{l}\text { The cow is located at the feeding zone with head } \\
\text { through the fence while searching, masticating or } \\
\text { sorting the feed. }\end{array}$ & $1550(27 \%)$ & Feeding & $1883(33 \%)$ \\
\hline $\begin{array}{l}\text { Feeding pattern } \\
\text { in concentrate } \\
\text { feeder }\end{array}$ & The cow has its head in the concentrate feeder. & $96(1.7 \%)$ & & \\
\hline $\begin{array}{l}\text { Feeding in } \\
\text { milking robot }\end{array}$ & $\begin{array}{l}\text { The cow has its head in the concentrates dispenser } \\
\text { in the milking robot. }\end{array}$ & $122(2.3 \%)$ & & \\
\hline Drinking & The cow is drinking water from the water trough. & $115(2 \%)$ & & \\
\hline $\begin{array}{l}\text { Standing in the } \\
\text { alleys }\end{array}$ & $\begin{array}{l}\text { The cow is standing in the alleys on at least three } \\
\text { legs with no movement to another place. }\end{array}$ & $1154(20 \%)$ & Standing & $1375(24 \%)$ \\
\hline $\begin{array}{l}\text { Standing in the } \\
\text { milking robot }\end{array}$ & $\begin{array}{l}\text { The cow is standing in the milking robot on at least } \\
\text { three legs }\end{array}$ & $52(1 \%)$ & & \\
\hline $\begin{array}{l}\text { Standing while } \\
\text { brushing }\end{array}$ & $\begin{array}{l}\text { The cow is standing at the cow brush on at least } \\
\text { three legs with no movement to another place. }\end{array}$ & $30(0.5 \%)$ & & \\
\hline Walking & $\begin{array}{l}\text { The cow is moving from one location to another by } \\
\text { moving more than } 2 \text { feet }\end{array}$ & $139(2.5 \%)$ & & \\
\hline Lying & $\begin{array}{l}\text { The cow is in a lying position (main body area } \\
\text { contact with floor) }\end{array}$ & $2502(43 \%)$ & Lying & $2502(43 \%)$ \\
\hline Total (SUM) & & & & $5760(100 \%)$ \\
\hline
\end{tabular}


537

538

539

540

541

542

543

544

545
Table 2. Precision ( $\mathrm{Pr}$ ) and sensitivity (Se) [\%] for each behavioural class and classification approach using different combinations of axes of the leg- and neck-mounted accelerometers ( $X Y Z, X Y, X Z, Y Z, X$, $Y$, and Z) and a sampling rate of $1 \mathrm{~Hz}$. K-NN: K-nearest neighbours, NB: Naïve Bayes, SVM: support vector machine. Values in blod indicate the highest values reached for each behaviour

\begin{tabular}{|c|c|c|c|c|c|c|c|c|c|c|c|c|c|c|c|c|}
\hline & & & \multirow{2}{*}{\multicolumn{2}{|c|}{ XYZ }} & \multirow{2}{*}{\multicolumn{2}{|c|}{$X Y$}} & \multirow{2}{*}{\multicolumn{2}{|c|}{$X Z$}} & \multirow{2}{*}{\multicolumn{2}{|c|}{ YZ }} & \multirow{2}{*}{\multicolumn{2}{|c|}{$x$}} & \multirow{2}{*}{\multicolumn{2}{|c|}{$Y$}} & \multirow{2}{*}{\multicolumn{2}{|c|}{ Z }} \\
\hline & & & & & & & & & & & & & & & & \\
\hline & & & $\mathrm{Pr}$ & $\mathrm{Se}$ & $\mathrm{Pr}$ & $\mathrm{Se}$ & $\mathrm{Pr}$ & $\mathrm{Se}$ & $\mathrm{Pr}$ & Se & $\operatorname{Pr}$ & $\mathrm{Se}$ & $\operatorname{Pr}$ & $\mathrm{Se}$ & $\mathrm{Pr}$ & Se \\
\hline \multirow{9}{*}{$\begin{array}{c}\text { Neck } \\
+ \\
\text { Leg }\end{array}$} & \multirow[t]{3}{*}{ K-NN } & Standing & 80 & 89 & 55 & 50 & 74 & 85 & 91 & 92 & 89 & 88 & 64 & 67 & 59 & 61 \\
\hline & & Feeding & 95 & 94 & 93 & 93 & 93 & 95 & 94 & 95 & 93 & 94 & 90 & 92 & 89 & 91 \\
\hline & & Lying & 97 & 94 & 84 & 86 & 97 & 94 & 99 & 100 & 99 & 99 & 90 & 88 & 89 & 89 \\
\hline & \multirow[t]{3}{*}{ NB } & Standing & 83 & 86 & 69 & 49 & 85 & 70 & 93 & 87 & 90 & 88 & 70 & 65 & 68 & 48 \\
\hline & & Feeding & 99 & 95 & 96 & 93 & 94 & 94 & 98 & 99 & 91 & 94 & 93 & 92 & 90 & 90 \\
\hline & & Lying & 96 & 96 & 76 & 91 & 91 & 91 & 99 & 95 & 99 & 99 & 85 & 91 & 80 & 95 \\
\hline & \multirow[t]{3}{*}{ SVM } & Standing & 94 & 96 & 83 & 76 & 88 & 90 & 96 & 96 & 90 & 91 & 82 & 75 & 80 & 72 \\
\hline & & Feeding & 98 & 99 & 95 & 97 & 95 & 96 & 98 & 98 & 95 & 94 & 93 & 95 & 92 & 93 \\
\hline & & Lying & 99 & 98 & 95 & 96 & 99 & 98 & 99 & 100 & 100 & 100 & 95 & 96 & 94 & 95 \\
\hline \multirow{9}{*}{ Leg } & \multirow[t]{3}{*}{ K-NN } & Standing & 63 & 52 & 37 & 51 & 47 & 48 & 47 & 54 & 56 & 42 & 41 & 55 & 54 & 40 \\
\hline & & & 82 & 81 & 70 & 61 & 65 & 66 & 66 & 62 & 65 & 68 & 59 & & 55 & 51 \\
\hline & & Lying & 96 & 97 & 80 & 88 & 97 & 95 & 99 & 100 & 98 & 100 & 92 & 88 & 87 & 87 \\
\hline & \multirow[t]{3}{*}{ NB } & Standing & 49 & 53 & 81 & 33 & 65 & 52 & 80 & 40 & 67 & 39 & 62 & 58 & 56 & 52 \\
\hline & & Feeding & 73 & 72 & 46 & 41 & 45 & 67 & 36 & 65 & 56 & 63 & 45 & 65 & 57 & 57 \\
\hline & & Lying & 97 & 93 & 69 & 95 & 88 & 99 & 99 & 99 & 100 & 98 & 70 & 83 & 85 & 96 \\
\hline & \multirow[t]{3}{*}{ SVM } & Standing & 76 & 68 & 40 & 49 & 47 & 56 & 36 & 59 & 48 & 63 & 48 & 59 & 59 & 50 \\
\hline & & Feeding & 81 & 86 & 68 & 66 & 64 & 91 & 65 & 89 & 65 & 87 & 68 & 82 & 56 & 62 \\
\hline & & Lying & 99 & 98 & 90 & 97 & 98 & 97 & 98 & 100 & 97 & 100 & 91 & 95 & 86 & 93 \\
\hline \multirow{9}{*}{ Neck } & \multirow[t]{3}{*}{ K-NN } & Standing & 63 & 52 & 53 & 40 & 41 & 53 & 54 & 61 & 58 & 64 & 55 & 56 & 46 & 55 \\
\hline & & Feeding & 88 & 96 & 92 & 95 & 93 & 93 & 91 & 94 & 89 & 93 & 91 & 93 & 87 & 92 \\
\hline & & Lying & 81 & 95 & 83 & 92 & 81 & 86 & 85 & 91 & 78 & 88 & 82 & 86 & 82 & 86 \\
\hline & \multirow[t]{3}{*}{ NB } & Standing & 66 & 43 & 46 & 52 & 35 & 42 & 63 & 56 & 62 & 56 & 59 & 58 & 46 & 59 \\
\hline & & Feeding & 84 & 95 & 95 & 95 & 92 & 95 & 96 & 95 & 88 & 89 & 91 & 92 & 82 & 87 \\
\hline & & Lying & 81 & 94 & 88 & 83 & 84 & 82 & 83 & 84 & 82 & 78 & 88 & 82 & 80 & 82 \\
\hline & \multirow[t]{3}{*}{ SVM } & Standing & 74 & 65 & 69 & 41 & 49 & 58 & 81 & 68 & 55 & 56 & 61 & 52 & 51 & 38 \\
\hline & & Feeding & 92 & 96 & 96 & 95 & 95 & 98 & 96 & 97 & 92 & 93 & 94 & 96 & 93 & 90 \\
\hline & & Lying & 83 & 97 & 83 & 94 & 78 & 94 & 82 & 95 & 78 & 96 & 83 & 93 & 79 & 96 \\
\hline
\end{tabular}


547 Table 3. Overall accuracy for each classification approach using different axes of the leg- and neck548 mounted accelerometers (XYZ, XY, XZ, YZ, X, Y, and Z) and a sampling rate of $1 \mathrm{~Hz}$. K-NN: K-nearest 549 neighbours, NB: Naïve Bayes, SVM: support vector machine. Values in blod indicate the highest 550 values for every approach.

\begin{tabular}{cllllllll} 
& & $X Y Z$ & $X Y$ & $X Z$ & $Y Z$ & $X$ & $Y$ & $Z$ \\
\hline Neck & K-NN & 93 & 81 & 92 & 97 & 95 & 85 & 84 \\
+ & NB & 93 & $\mathbf{7 7}$ & 90 & 97 & 95 & 85 & $\mathbf{7 6}$ \\
Leg & SVM & $\mathbf{9 8}$ & $\mathbf{9 3}$ & $\mathbf{9 6}$ & $\mathbf{9 9}$ & $\mathbf{9 7}$ & $\mathbf{9 3}$ & $\mathbf{9 1}$ \\
\hline \multirow{4}{*}{ Leg } & K-NN & 84 & 69 & 76 & 82 & 82 & 72 & 68 \\
& NB & 83 & 68 & 78 & 78 & 75 & 75 & 67 \\
& SVM & $\mathbf{8 8}$ & $\mathbf{8 0}$ & $\mathbf{8 4}$ & $\mathbf{8 6}$ & $\mathbf{8 5}$ & $\mathbf{7 9}$ & $\mathbf{7 8}$ \\
\hline \multirow{3}{*}{ Neck } & K-NN & 86 & 82 & $\mathbf{7 8}$ & 81 & 78 & 80 & 78 \\
& NB & 84 & $\mathbf{7 8}$ & $\mathbf{7 9}$ & 82 & 76 & 82 & 76 \\
& SVM & $\mathbf{9 2}$ & $\mathbf{8 6}$ & $\mathbf{8 4}$ & $\mathbf{8 4}$ & $\mathbf{8 3}$ & $\mathbf{8 5}$ & $\mathbf{8 2}$ \\
\hline
\end{tabular}

551 